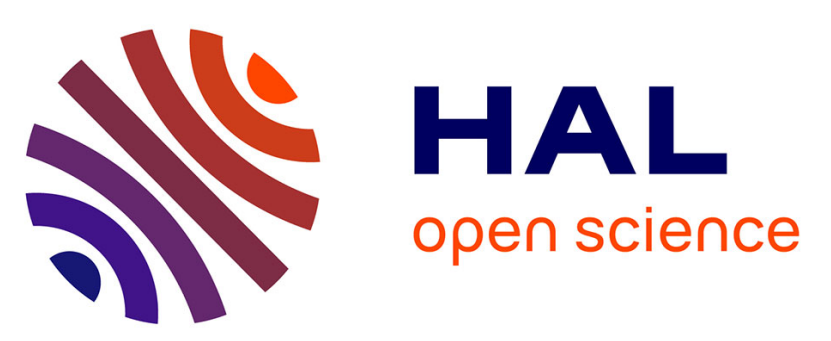

\title{
FIRST RESULTS FROM DELCO AT PEPCalifornia Institute of Technology-Stanford Linear Accelerator Center and Stanford University Collaboration
}

J. Kirkby, W. Atwood, P. Baillon, B. Barish, G. Bonneaud, H. Destaebler, G. Donaldson, R. Dubois, M. Duro, S. Gao, et al.

\section{To cite this version:}

J. Kirkby, W. Atwood, P. Baillon, B. Barish, G. Bonneaud, et al.. FIRST RESULTS FROM DELCO AT PEPCalifornia Institute of Technology-Stanford Linear Accelerator Center and Stanford University Collaboration. Journal de Physique Colloques, 1982, 43 (C3), pp.C3-45-C3-51. 10.1051/jphyscol:1982308 . jpa-00221861

\section{HAL Id: jpa-00221861 https://hal.science/jpa-00221861}

Submitted on 1 Jan 1982

HAL is a multi-disciplinary open access archive for the deposit and dissemination of scientific research documents, whether they are published or not. The documents may come from teaching and research institutions in France or abroad, or from public or private research centers.
L'archive ouverte pluridisciplinaire HAL, est destinée au dépôt et à la diffusion de documents scientifiques de niveau recherche, publiés ou non, émanant des établissements d'enseignement et de recherche français ou étrangers, des laboratoires publics ou privés. 
JOURNAL DE PHYSIQUE

Colloque C3, supplément au no 12, Tome 43, décembre 1982

page $\mathrm{C} 3-45$

\section{FIRST RESULTS FROM DELCO AT PEP*}

California Institute of Technology-Stanford Linear Accelerator Center and Stanford University Collaboration

Presented by J. Kirkby

SLAC, P.O. Box 4349, Stanford, CA 94305, U.S.A.

1. Introduction - The data I will discuss was recorded by the DELCO detector at the PEP $\mathrm{e}^{t} \mathrm{e}^{-}$storage ring. The sample corresponds to an integrated luminosity of $22 \mathrm{pb}^{-1}$ at $\mathrm{Ecm}=29 \mathrm{GeV}$. The data was accumulated over a recent period (FebruaryJune 1982) and so these results should be considered to be preliminary.

Since the original SPEAR detector[2] has been modified to operate at higher energies we will provide a brief technical description. The detector (Figs. 1 and 2) has an atmospheric-pressure threshold gas Cerenkov counter of 36 cells covering a total of $60 \%$ of $4 \pi$ steradians. An open-geometry magnet provides a field integral of $1.8 \mathrm{kG}-\mathrm{m}$ to measure the momenta of charged particles, while maintaining a low mass

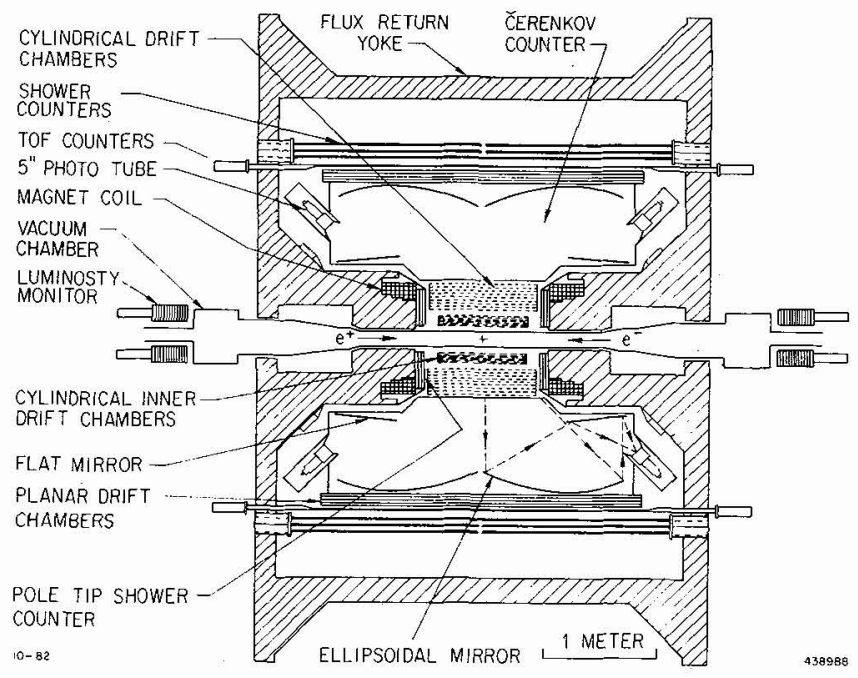

Fig. 1: Polar view of DELCo 
$\left(0.05 \mathrm{x}_{0}\right)$ between the interaction point and the Cerenkov gas radiator. Charged particles are tracked between radii of 12 and $50 \mathrm{cms}$ in a 16-layer cylindrical drift chamber system before entering the Cerenkov counter. There are 6 layers of planar drift chambers beyond the counter, at an average radius of about $170 \mathrm{~cm}$. Tracks with $|\cos \theta|<0.65$ traverse the full 22 drift chamber layers and have a momentum resolution, $\sigma_{\mathrm{p}} / \mathrm{p}=\sqrt{[0.025 \mathrm{p}(\mathrm{GeV} / \mathrm{c})]^{2}+0.06^{2}}$. Other components of DELCO include timeof-flight counters, luminosity monitors and a $\mathrm{Pb}$-scintillator shower counter system covering the central and forward (pole-tip) regions.

The Cerenkov counter is filled with isobutane and gives an average signal of 17 photo-electrons (p.e.) with a time resolution of $300 \mathrm{ps, \text {for }}$ $\beta=1$ particles. (The pulse height corresponds to a mean quality factor, $\left\langle\mathrm{N}_{\mathrm{o}}\right\rangle=80 \mathrm{~cm}^{-1}$.) This counter provides

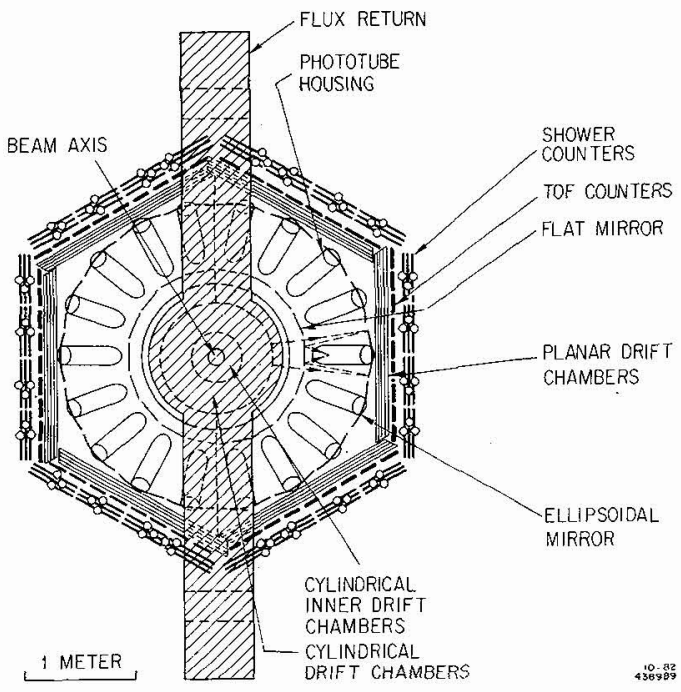

Fig. 2: Azimuthal view of DELCO. e/hadron discrimination below $\pi$ threshold $(2.7 \mathrm{GeV} / \mathrm{c})$ and, at higher energies, distinguishes $\pi$ from $\mathrm{Kp}$. On1y studies involving $\pi / \mathrm{Kp}$ identification are discussed here.

2. Cabibbo Angle in $T$ Decays - Although all present experimental measurements have served to reinforce our picture of $e-\mu-\tau$ universality, it is of recognized importance to refine this study either by new approaches or by repeating previous measurements with better precision. A fundamental component of lepton universality is a common Cabibbo angle, $\theta_{c}$. For the $\tau$ this implies the relative decay rates, $\tau^{-} \rightarrow \nu_{\tau} \overline{u s} / \tau^{-} \rightarrow \nu_{\tau} \overline{u d}$ are equal to $\tan ^{2} \theta_{c}$ after accounting for phase-space differences. The measurement is made by comparing the relative branching ratios to $v_{\tau} K^{-} n \pi$ and $v_{\tau} \pi^{-} n \pi .[3]$ Previous measurements[4] have isolated the $\nu_{\tau} K^{-}$and $v_{\tau} K^{*-}$ decays. In this study we isolate inclusive one-prong (IP) decays of the type $\nu_{\tau} \mathrm{K}^{-} \mathrm{n} \pi_{0}, \mathrm{n} \geq 0$.

We select $\tau^{+} \tau^{-}$decays in the $1 P-1 P$ and $1 P-\mathbb{P}$ (multi-prong) topologies, where the prong count is made within each hemisphere centered on the thrust axis. The major backgrounds to the $1 P-1 P$ topology are from QED sources and these are excluded primarily by cutting on thrust $(<0.96)$, acoplanarity $(\Delta \phi>15 \mathrm{mr})$, acolinearity $(\cos \Delta \theta<0.998)$ and total charged particle momentum $(>5 \mathrm{GeV} / \mathrm{c})$, in addition to rejecting all e-e events. The $1 P-\mathbb{P}$ events are initially contaminated by radiated QED events and hadronic events - these are removed primarily by requiring low MP invariant mass $\left(<2.5 \mathrm{GeV} / \mathrm{c}^{2}\right)$ in addition to the previous thrust cut.

There are $4671 \mathrm{P}-1 \mathrm{P}$ and $1261 \mathrm{P}-\mathrm{MP}$ events which satisfy the cuts. After correcting by the detection efficiencies, we measure the total cross section ratio $R_{T} \bar{\tau}=1.0 \pm .06 \pm .3$, where the systematic error reflects our uncertainty in luminosity (10\%), detection efficiency (5\%) and background contamination (20\%).

We now look at the Cerenkov response (Fig.3) of the $9051 \mathrm{P}$ tracks which remain after excluding those with $\cos \theta<0.06$ (since they pass close to a mirror boundary at $\theta=90^{\circ}$ ). The tracks display two clear Cerenkov thresholds: one has occurred at very low momentum and corresponds to electrons and the other occurs between 2 and $3 \mathrm{GeV} / \mathrm{c}$, corresponding to $\mu$ and $\pi$. Beyond these thresholds only a few tracks fail to produce a strong Cerenkov response - these are potential $\mathrm{K}^{-}$. (Since the $\tau$ does not decay to a proton there is no $\mathrm{Kp}$ Cerenkov ambiguity for a clean sample of $\tau$ decays.) The candidate $\mathrm{K}^{-}$prongs are required to have a Cerenkov pulse height below 3 p.e. and a momentum above $4.5 \mathrm{GeV} / \mathrm{c}$. The latter cut is made conservatively in order to avoid sensitivity to 'tails' in the momentum distribution. For the same reason we do not make a 
high-momentum cut and account for the $\mathrm{K}$ inefficiency above $\mathrm{K}$ threshold $(9.4 \mathrm{GeV} / \mathrm{c})$ with an appropriate correction to the detection efficiency. Each of the 13 events which survive these requirements was hand-scanned and two were rejected for reasons of an extra track and a mis-track which incorrectly passed the $\cos \theta$ cut.

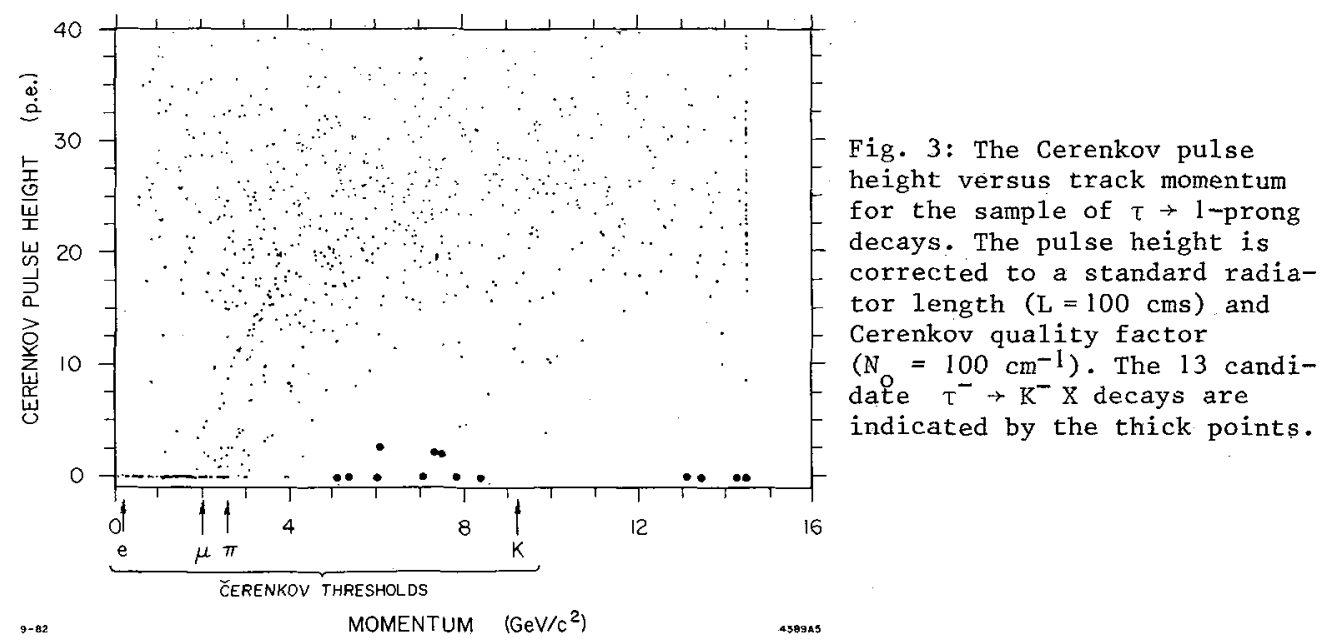

Since the final $K$ candidates form only $1 \%$ of the full detected $1 \mathrm{P}$ sample we have investigated whether they are falsely generated by instrumental effects. We selected a sample of Bhabha events independently of the Cerenkov counter and then, after requiring the same geometrical constraints as the $\mathrm{K}$ candidates, asked how often the Cerenkov response was below $3 \mathrm{p} . e$. No examples were found in 2000 Bhabha events and so the Cerenkov inefficiency is $<0.610^{-3}(95 \% \mathrm{CL})$. We also found no indication of a clustering of the events near to mirror-edges. Finally, gross mis-measurement of the tracks with momentum below $\pi$ threshold was excluded by investigating the track-quality of each $\mathrm{K}$ candidate. Our conclusion is that the 11 events are genuine. equation:

The signal events $\left(\mathrm{N}_{\mathrm{K}}\right)$ are used to measure the Cabibbo angle according to the

$$
\mathrm{N}_{K}=\mathrm{N}_{\tau \bar{\tau}}(4 \pi) \times \tan ^{2} \theta_{c} \times 2\left[\varepsilon\left(\mathrm{K}^{-}\right) \mathrm{b}\left(\pi^{-}\right) \rho+\frac{1}{3} \varepsilon\left(\mathrm{K}^{-} \pi^{0}\right) b\left(\pi^{-} \pi^{0}\right) \rho^{\prime}+f \varepsilon\left(\mathrm{K}^{-} \mathrm{n} \pi^{0}\right) \mathrm{b}\left(\pi^{-} \mathrm{n} \pi^{0}\right) \rho^{\prime \prime}\right]
$$

In this expression, the total $\tau \bar{\tau}$ sample over $4 \pi$ steradians, $N_{\tau} \bar{\tau}(4 \pi)$, is determined from the luminosity measurement and the $\tau^{-} \rightarrow K^{-} X$ detection efficiencies, $\varepsilon\left(K^{-} X\right)$ by Monte Carlo. The square parentheses contain the non-strange hadronic branching ratios, $b\left(\pi^{-} \mathrm{X}\right)$, corrected for relative phase space, $\rho$, and appropriate one-prong- $\mathrm{K}$ branching ratio, $f$, e.g., $b\left(K^{*}-\rightarrow K^{-} \pi^{0}\right)=1 / 3$. The result is the measurement $\tan ^{2} \theta_{c}=0.08 \pm 0.03 \pm 0.04$, which is equivalent to the branching ratio, $\mathrm{b}\left(\tau^{-} \rightarrow \mathrm{K}^{-}+\right.$ neutrals $)=0.02 \pm 0.007 \pm 0.01$. This value, albelt with large errors, is consistent with Cabibbo angle measurements in other processes. With further analysis and a larger data sample we hope to improve the precision substantially and to extend the analysis to the multi-prong $t$ decays, for which there are no previous measurements.

3. Charged Particle Fractions at Intermediate $x$ - Our present understanding of the materialization of hadrons from quarks is based on simple models. A major experimental task is to establish detailed information which will allow these models to be transformed into a theoretical framework. In these studies, $e^{+} e^{-}$annihilation is particularly important since it provides a clean, mono-energetic source of each quark. Furthermore, in certain cases, the flavour of the parent quark can be identified. In this section we present data on the inclusive charged particle fractions at intermediate $x \quad(0.20<x<0.55)$ and the following section deals with the fragmentation of charmed quarks.

Such measurements frequently must be interpreted through Monte Carlo calculations which model the fragmentation process. We use a Monte Carlo, written by H. Yamamoto in our collaboration, based on Feynman-Field fragmentation with first- 
order QCD effects included. The adjustable parameters have been chosen to fit closely the data[5] from MARK II and TASSO as follows: mean $p_{t}$ of sea quark, $\sigma_{q}=$ $0.35 \mathrm{GeV} / \mathrm{c}$, strong coupling constant, $\alpha_{\mathrm{s}}=0.21$, thrust cutoff $=0.96$, pseudoscalar: vector meson ratio $=0.6: 0.4$ and $\alpha_{F}=0.75$ (uds), 0.2 (c) and -0.4 (b). (The Feynman-Field quark fragmentation function is $1-\alpha_{F}+3 \alpha_{F}(1-x)^{2}$.) In this version of the program, baryons are not generated.

The sample of hadronic events is selected by cuts which are chosen to exclude background contributions from cosmics, two-photon processes, beam-gas interactions, QED and $\tau$ events. The primary cuts require at least five tracks emerging from a region within the interaction volume $(|\langle z\rangle|<4.5 \mathrm{~cm})$. Furthermore the total charged and neutral energy must exceed $8.5 \mathrm{GeV}$ and not display a strong asymmetry along the beam direction || $\mathrm{E}_{\mathrm{ASYM}} / \mathrm{E}_{\mathrm{TOT}} \mid<0.35$,

where $\mathrm{E}_{\mathrm{ASYM}}=\sum|\overrightarrow{\mathrm{P}}| \cos \theta+\sum \mathrm{E}_{\text {NEUTRAL }} \cos \theta$ and $\left.\mathrm{E}_{\mathrm{TOT}}=\sum|\overrightarrow{\mathrm{P}}|+\sum \mathrm{E}_{\text {NEUTRAL }}\right)$. There are 8050 events which survive these cuts. After accounting for the detection efficiency this provides a measurement of the hadronic cross section ratio, $\mathrm{R}_{\mathrm{HAD}}=$ $4.0 \pm 0.6$. The error is dominated by systematic uncertainties in the luminosity $(10 \%)$, detection efficiency ( $10 \%)$ and background (5\%).

We have made detailed comparisons between the experimental hadronic events and a sample generated by our Monte Carlo. In all distributions the agreement is excellent which therefore implies consistency between our data and TASSO/MARK II. One example is the inclusive charged particle cross section versus $x$ as shown in Fig. 4 .

We analyze the particle fractions in this distribution according to the response of the associated Cerenkov, without regard to extra tracks which may enter the same cell. This avoids possible 'bias' in measuring high momentum tracks since they are often in multiply-occupied cells - although it necessitates a correction be made for cases where the cells involve either two high momentum $(>2.7 \mathrm{GeV} / \mathrm{c})$ tracks or an associated electron track. The particle-type is described as 'heavy' if it produces $<2$ p.e. Cerenkov pulse height.

The data (Fig. 5) indicate a heavy fraction of about $80 \%$ below $\pi$ threshold. This fraction, which nominally measures $\pi \mathrm{Kp}$, is artificially reduced by high momentum $\pi$ 's and, to a lesser extent, electrons (such as from $\gamma$ conversions) which may accompany other hadrons. These processes are well explained at low energies by the Monte Carlo calculation, as indicated by the smooth curve. We therefore have confidence in correctly accounting for them at higher energies.

We restrict our measurement of the $\pi / \mathrm{Kp}$ fraction to a reduced region between the $\pi$ and $K$ thresholds in order to avoid sensitivity to the momentum resolution. The result (Fig. 6) shows that the $\pi$ fraction in the region $0.2<x<0.55$ is

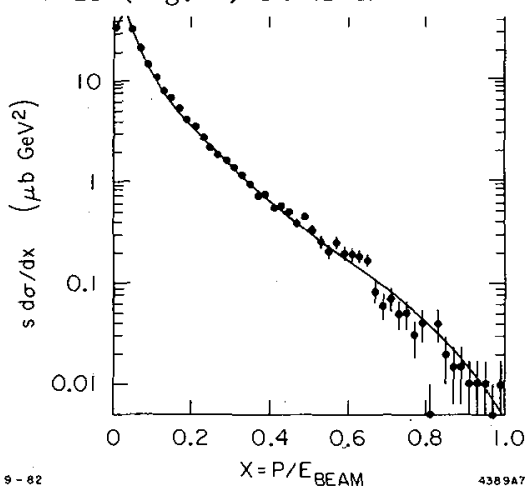

Fig. 4: The inclusive cross section $s \mathrm{do} / \mathrm{dx}$ versus $\mathrm{x}$ for charged particles in hadronic annihilation events. The smooth curve indicates the Monte Carlo distribution. Systematic errors in the vertical scale of $\pm 15 \%$ are not included in the error bars.

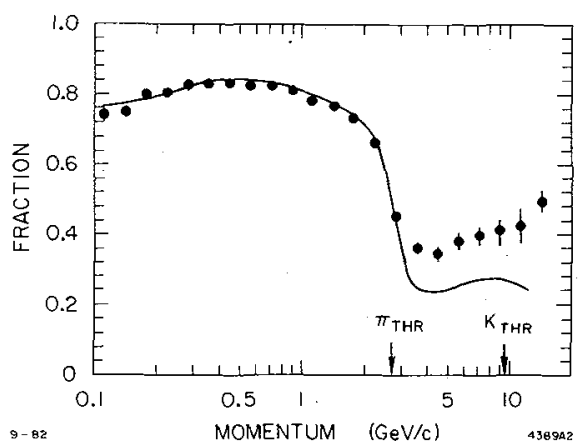

Fig. 5: The fraction of tracks which have $<2$ p.e. Cerenkov pulse height versus momentum. The analysis allows multiple tracks into a Cerenkov cell. The smooth curve indicates the Monte Carlo distribution. 
approximately constant and lies between 0.6 and 0.65 , with an estimated systematic error of \pm 0.1 . [6] This value is not anticipated in our Monte Carlo calculation (as indicated in Figs. 5 and 6) and the difference may be attributed to protons in the experimental data. We are proceeding to investigate the characteristics and sources of this large signal of heavy hadrons and to make an experimental measurement of the proton fraction at high $x(>0.8)$.

4. Charged D* Production - As we have indicated, this study is important to explore the properties of the fragmentation of heavy quarks. In addition, the identification of $\mathrm{D}^{*}$ decays provides a valuable experimental tool for 'flavourtagging' hadronic events.

The $\mathrm{D}^{*+}$ are selected by means of the decays $\mathrm{D}^{*+} \rightarrow \mathrm{D}^{0} \pi^{+}$followed by $\mathrm{D}^{0} \rightarrow \mathrm{K}^{-} \pi^{+}$. The low $Q$ in the $\mathrm{D}^{*+}$ decay results in the distinct signature $[7,8]$ of a $D^{0}$ and $\pi^{+}$ with almost identical $\beta$ and direction.

We require at least one prong have momentum

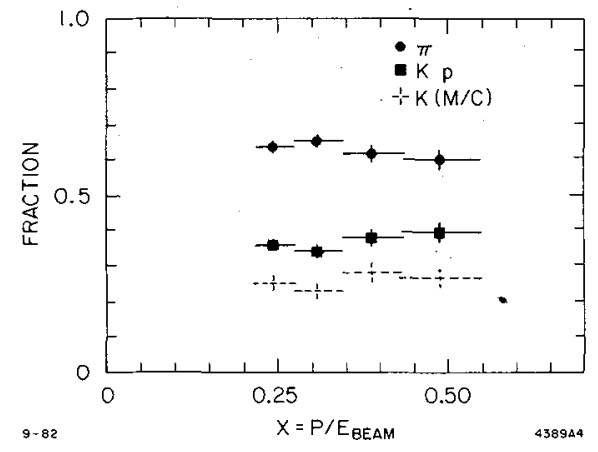

Fig. 6: The $\pi$ and $\mathrm{kp}$ chargedparticle fractions versus $x$ in the range $.2<\mathrm{x}<.55$. The Monte Carlo prediction of the $\mathrm{K}$ fraction is also indicated. The vertical error bars do not include an estimated systematic error of \pm 0.1 on each point. above $2.7 \mathrm{GeV} / \mathrm{c}$ such that it is identifiable as $\pi$ (Cerenkov pulse) or $\mathrm{K}$ (no pulse)。 The Cerenkov pulse corresponds to at least 4 p.e. produced within $1.2 \mathrm{~ns}$ of the nominal event time. A second prong is selected if it has opposite charge to the first and lies within an opening angle of $45^{\circ}$. No particle identification is required for this prong; it is labeled $\pi$ or $\mathrm{K}$ according to the identity of the first. A candidate $\mathrm{D}^{0}$ is accepted if the $\mathrm{K} \pi$ invariant mass lies in the range $1.5-2.5 \mathrm{GeV} / \mathrm{c}^{2}$. The $\mathrm{D}^{0}$ momentum, $\overrightarrow{\mathrm{P}}_{\mathrm{D}} 0$, is computed as the vector sum of the measured particle momenta and the $\mathrm{D}^{0}$ energy $\mathrm{D}^{0}$ kinematically constrained to the $\mathrm{D}^{0}$ mass by the equation,

$$
\mathrm{E}_{\mathrm{D}^{0}}=\left(\mathrm{M}_{\mathrm{D}^{0}}^{2}+\left|\overrightarrow{\mathrm{P}}_{\mathrm{D}^{0}}\right|^{2}\right)^{1 / 2}
$$

For those events which contain a candidate $\mathrm{D}^{0}$, the $\mathrm{D}^{0}$ and any remaining charged particle (assumed to be $a \pi$ ) of opposite-sign to the $K$ are paired. The $D^{0} \pi^{+}-D^{0}$ mass difference spectrum (Fig.7a) shows a clear signal of $\mathrm{D}^{*^{+}}$decays. Events which involve $K$ identification emphasize the signal region and indicate a stronger association of charm with strange rather than non-strange high-energy particles. We measure the background in this data by taking the same $D^{0}$ candidates but pairing them with $\pi^{\prime}$ s having the same sign as the $\mathrm{K}$ (Fig. $7 \mathrm{~b}$ ). These cannot result from $\mathrm{D}^{*+}$ decay and so measure the distribution due to chance combinations. We thereby estimate a background of 6 events in the $25 \mathrm{D}^{*}$ candidates observed below $155 \mathrm{MeV} / \mathrm{c}^{2}$ in Fig. $7 \mathrm{a}$.

We calculate the detection efficiency to be roughly constant (at 29\%) for $D^{*}$ momenta above $6 \mathrm{GeV} / \mathrm{c}$ and to fall at lower momenta. We therefore restrict our measurements to $z>0.3$, where $z=E_{D *+} / E_{B E A M}$. After correcting by the branching ratios, [9] we find the inclusive cross section ratio, $R\left(e^{+} e^{-} \rightarrow D^{* \pm} X\right)=(2.0 \pm 0.6 \pm 0.7)$ in the $\mathrm{z}$ range above 0.3 . The systematic error is due to uncertainties in the $\mathrm{D}$ branching ratios (26\%), luminosity $(10 \%)$ and detection efficiency $(20 \%)$. Since the total cross section ratio for a11 charm and bottom quarks is estimated to be 3.7 at this energy, we find that heavy quark fragmentation is dominated by $D^{*}$ production (after making the assumption that the neutral and charged $D^{*}$ cross sections are approximately equal).

The $\mathrm{D}^{*+}$ differential cross section $\mathrm{s} d \sigma / \mathrm{dz}$ (Fig.8) indicates that the fragmentation of heavy quarks occurs predominately at large values of $z$ (the mean value is $0.6 \pm 0.1)$ as had been anticipated from kinematic arguments. This distribution provides a measure of the charmed quark fragmentation function since a Monte Carlo calculation indicates that almost all $(90 \%)$ of the $\mathrm{D}^{*+}$ in our acceptance are due to a primary $c$ rather than $a$ b quark. 

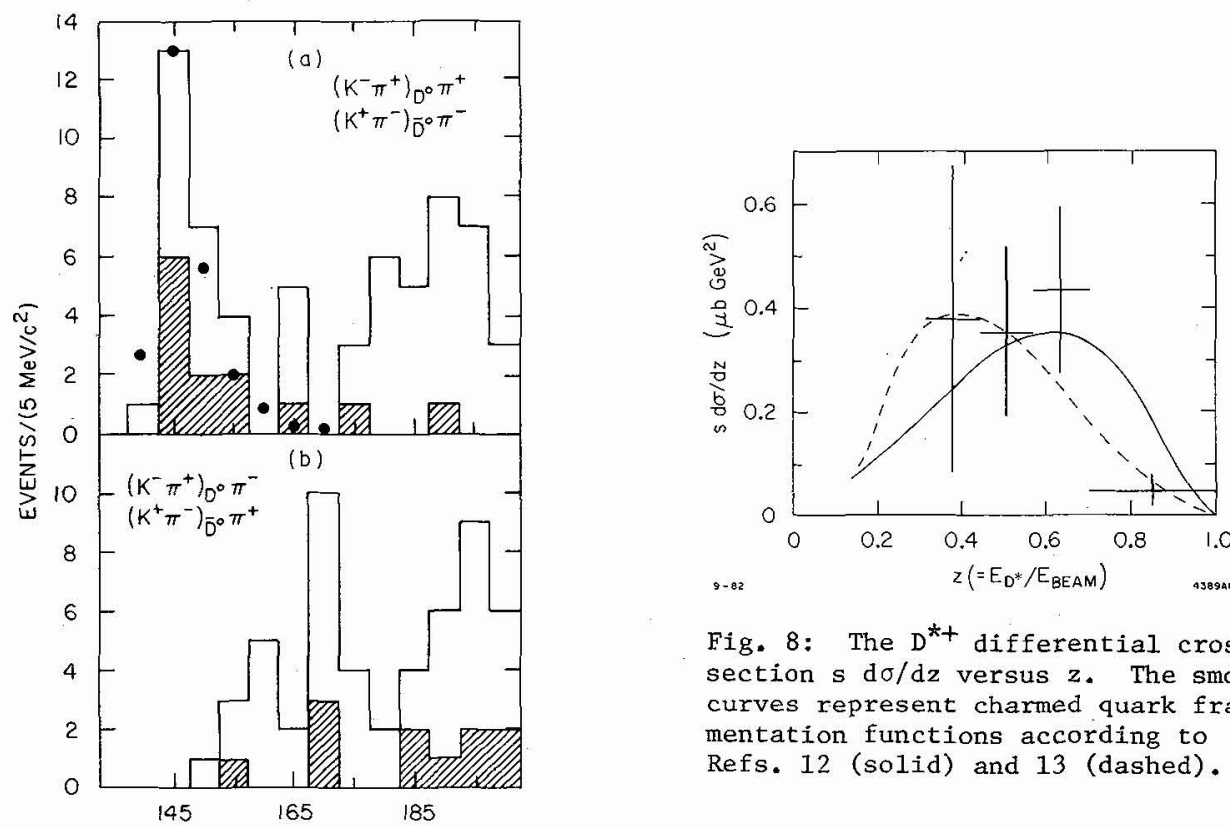

Fig. 8: The $D^{*+}$ differential cross section $s \mathrm{~d} \sigma / \mathrm{d} z$ versus $z$. The smooth curves represent charmed quark fragmentation functions according to Refs. 12 (solid) and 13 (dashed).

${ }_{4389 \mathrm{AB}}^{9-22} \quad \mathrm{D}^{\circ} \pi^{ \pm} 0^{\circ}$ MASS DIFFERENCE $\left(\mathrm{MeV}^{2} \mathrm{C}^{2}\right)$

Fig. 7: a) The $\mathrm{D}^{0} \pi^{+}-\mathrm{D}^{0}$ mass dif-

ference. The dots outline the distribution predicted by Monte Carlo for the process $\mathrm{D}^{*+} \rightarrow \mathrm{D}^{0} \pi^{+}$.

b) The $\mathrm{D}^{0} \pi^{-}-\mathrm{D}^{\mathrm{O}}$ mass difference.

Events which involve a Cerenkov-

identified $\mathrm{K}$ are indicated by the

shading.

These data are consistent with other measurements from $e^{+} e^{-}$annihilation $[8,10]$ and $\nu$ interactions. [11] If the charm fragmentation function is expressed [12] in the form $1 / z\left[1-z^{-1}-\varepsilon(1-z)^{-1}\right]^{2}$, all data are found to be consistent with the parameter $\varepsilon=0.25$. However, the accuracy of the present data is not sufficient to distinguish between alternative predictions, such as that described in Ref. 13. Both of these distributions are indicated in $\mathrm{Fig} .8$.

5. Sunmary - The $\tau$ lepton decays to $\mathrm{K}^{-}+$neutrals with a branching ratio of about 2\%, consistent with the value expected from the standard Cabibbo angle.

A large fraction, approximately $40 \%$ of the prongs at medium $x(.2<\mathrm{x}<.55)$ are found to be $\mathrm{K}+\mathrm{p}$.

A large $D^{* \pm}$ signal is observed, indicating that $D^{*}$ production may saturate the total charm cross section. In contrast with the lighter quarks, charmed quarks fragment predominantly at large values of $z$. (The striking difference in the fragmentation of heavy and light quarks is seen by comparing Figs. 4 and 8; D* production exceeds $\pi+k+p$ for $x>0.5$ !)

\section{REFERENCES}

[1] Where two errors are quoted in the text, the first is statistical and the second is the estimated systematical error.

[2] BACINo (W.) et a1., Phys. Rev. Lett., 1978, 40,671.

[3] Throughout, in order to avoid awkward notation, we will refer only to one charge conjugate state but imply both states. 
[4] DORFAN (J.) et a1., Phys. Rev. Lett., 1981, 46, 215; BLOCKER (C. A.) et al., Phys. Rev. Lett., 1982, 48, 1586.

[5] See, for example, the review paper of MESS (K. H.) and WIIK (B. H.), DESY 82/1] and references contained therein.

[6] This is consistent with TASSO measurements as reported by LüKE (D.) at this conference.

[7] FITCH (V. L.) et al., Phys. Rev. Lett., 1981, 46, 761.

[8] YELTON (J.) et al., Phys. Rev. Lett., 1982, 49, 430.

[9] We use the branching ratios, $\mathrm{b}\left(\mathrm{D}^{*^{+}} \rightarrow \mathrm{D}^{0} \pi^{+}\right)=(0.64 \pm 0.11)$ and $\mathrm{b}\left(\mathrm{D}^{0} \rightarrow \mathrm{K}^{-} \pi^{+}\right)=$ $(0.024 \pm 0.004)$.

[10] See the reports from TRILLING (G.) and LÜKF (D.) at this conference.

[11] ABRAMOVICZ (H.) et a1., CERN EP/82-77 (submitted to PHYSIK).

[12] PETERSON (C.) et al., SLAC-PUB-2912 (1982), and SCHLATTER (D.), Proceedings of the 1982 SLAC Summer School (to be published).

[13] GOTTSCHALK (T. D.), Caltech preprint (in preparation).

*W.B. Atwood, P.H. Baillon, B.C. Barish, G.R. Bonneaud, H. DeStaebler, G.J. Donaldson, R. Dubois, M.M. Duro, S.G. Gao, Y.Z. Huang, G.M. Irwitn,

R.P. Johnson, H. Kichimi, J. Kirkby, D.E. Klem, D.E. Koop, J. Ludwig, G.B. Mills, A. Ogawa, D. Perret-Gallix, R. Pitthan, D.L. Pollard, C.Y. Prescott, L.Z. Rivkin, L.S. Rochester, W. Ruckstuh1, M. Sakuda, S.S. Sherman, E.J. Siskind, R. Stroynowski, S.Q. Wang, S.G. Wojcicki, H. Yamamoto, W.G. Yan and C.C. Young.

Work supported by the Department of Energy, contract numbers DE-ACO3-76SF00515 and DE-AC03-81-ER40050, and The Nationa1 Science Foundation. 